\title{
Hydration layers at the graphite-water interface: Attraction or confinement
}

\author{
Hagen Söngen, ${ }^{1, *}$ Ygor Morais Jaques $\odot,{ }^{2}$ Lidija Zivanovic, ${ }^{2}$ Sebastian Seibert, ${ }^{1}$ Ralf Bechstein, \\ Peter Spijker, ${ }^{2}$ Hiroshi Onishi, ${ }^{3}$ Adam S. Foster, ${ }^{2,4,5}$ and Angelika Kühnle ${ }^{1}$ \\ ${ }^{1}$ Physical Chemistry I, Bielefeld University, Universitätsstraße 25, 33615 Bielefeld, Germany \\ ${ }^{2}$ COMP Centre of Excellence, Department of Applied Physics, Aalto University, Helsinki FI-00076, Finland \\ ${ }^{3}$ Department of Chemistry, Kobe University 1-1 Rokkodai-cho, Nada-ku, Kobe, Hyogo 657-8501, Japan \\ ${ }^{4}$ Graduate School Materials Science in Mainz, Staudingerweg 9, 55128, Germany \\ ${ }^{5}$ Division of Electrical Engineering and Computer Science, Kanazawa University, Kanazawa 920-1192, Japan
}

(Received 10 July 2019; revised manuscript received 27 August 2019; published 8 November 2019)

\begin{abstract}
Water molecules at solid surfaces typically arrange in layers. The physical origin of the hydration layers is usually explained by two different reasons: (1) the attraction between the surface and water and (2) the water confinement due to the surface. While the attraction is specific to the particular solid, the confinement is a general property of surfaces; a differentiation between the two effects is, therefore, critical for research on interactions at aqueous interfaces. Here, we investigate the graphite-water interface, which is a widely used model system where the solid-water attraction is often considered to be negligible. Similar to previous studies, we observe hydration layers using three-dimensional atomic force microscopy at the graphite-water interface. We explain why the confinement could cause the formation of hydration layers even in the absence of attraction between surface and water by employing Monte Carlo simulations. Using additional molecular dynamics simulations, we continue to show that at ambient conditions, however, the confinement alone does not cause the formation of layers at the graphite-water interface. We thereby demonstrate that there is a significant graphite-water attraction.
\end{abstract}

DOI: 10.1103/PhysRevB.100.205410

Despite the importance of liquid water, surprisingly little is known about water and its interactions on a molecular level. For example, the mechanisms of water evaporation [1] and the melting of ice [2] have only recently been understood and many other questions, such as the interfacial structure of water at solid surfaces, is still being investigated [3]. With improvements of experimental techniques it has become possible to image solid-water interfaces with unprecedented detail [4]. A common observation at these interfaces is the layered arrangement of water: Hydration layers have been observed at a large variety of solid-water interfaces, including mineral-water interfaces [5-7], graphite-water [8], and metalwater interfaces $[9,10]$. Layers at solid-water interfaces have been observed using X-ray reflectivity measurements [11-13], the surface force apparatus [14-17], and with high-resolution three-dimensional (3D) atomic force microscopy (AFM)[5].

A possible origin for layers are attractive interactions between the liquid molecules and the surface, giving rise to preferred positions for the liquid molecules and, consequently, to local maxima in the liquid density. Another origin for the layering of the liquid is the presence of the surface itself: Since the liquid molecules cannot penetrate the solid surface, their positions are vertically confined (referred to

\footnotetext{
*hagen.soengen@uni-bielefeld.de
}

Published by the American Physical Society under the terms of the Creative Commons Attribution 4.0 International license. Further distribution of this work must maintain attribution to the author(s) and the published article's title, journal citation, and DOI. as confinement in this work). It is desirable to differentiate between layering through attraction and layering through confinement: While the attraction specifically results from the interaction between the liquid molecules and the surface, liquid confinement would be generally expected for any solid surface.

Many theoretical studies have addressed the structure of solid-liquid interfaces with a particular focus on the formation of solvation layers. Starting with early works by Fisher and Widom [18], Abraham [19-22], Henderson [23,24], Snook [25-27], van Megen [28,29], and others, various approximative analytical approaches, Monte Carlo (MC) and molecular dynamics (MD) simulations, have been employed. Different models for the molecule-wall and molecule-molecule interaction have been investigated, including hard walls and hard spheres, Lennard-Jones models with different powers and purely repulsive Lennard-Jones models using the WeeksChandler-Andersen (WCA) cutoff [30]. These different models cover a large parameter space, especially when additionally considering the surface structure, the system size, the average liquid density, and temperature. For example, Henderson, Abraham, and Barker [23] as well as Snook and van Megen [25] have found a layering of a hard-sphere liquid in the presence of hard walls in three dimensions. MC simulations using different combinations of purely repulsive wall interactions with different liquid-liquid interactions (such as hard spheres and Lennard-Jones interactions) all result in layering [26-28]. The same applies for simulations by Abraham et al. [20-22] for various combinations of hardsphere fluids in contact with purely repulsive Lennard-Jones walls. Recent MD simulations by different authors have also 
reported that layering is obtained for water between purely repulsive walls [31-33].

Strikingly, other research papers exist that have reported seemingly contradicting results: In these studies, no layers have been observed in the presence of purely repulsive confining walls. This has been claimed in one of the above-cited publications by Abraham [20] where a Lennard-Jones liquid in contact with a hard wall has been used. Only when Abraham additionally considered attractive interactions between the liquid molecules and the wall (again using a LennardJones model), he observed the layers for this system. The author has argued that the discrepancy to the publication by Snook [26], in which layering has been observed for a similar system with a hard wall, emerges from a smaller average bulk density. Moreover, recent MD simulations by Willard and Chandler [34] and by Choudhury [35] have shown that layering at a generic interface and at the graphite-water interface, respectively, scales with the degree of the attraction. However, it is left open whether the layering completely vanishes when considering no attraction. Mozaffari has claimed that for vanishing attraction, no layering can be observed at the graphite-water interface [36], although some of the vertical density profiles shown in Ref. [36] exhibit a notable oscillatory behavior. Experimental water contact angle measurements recently prompted the following question: "Are graphitic surfaces hydrophobic" [37]?

From the above-mentioned literature, it is difficult to draw a clear conclusion about the influence of attractive interactions and confinement on the manifestation of hydration layers at the graphite-water interface. Moreover, a comprehensible explanation of layer formation in the absence of attraction, i.e., by confinement alone, is lacking so far. Here, we provide this explanation. Furthermore, we explore how the surfacemolecule and molecule-molecule interactions impact the hydration structure.

We start by reporting high-resolution 3D AFM data at the graphite-water interface that exhibit a layered arrangement of interfacial water, extending approximately one nanometer above the surface. Next, we use MC simulations to demonstrate that confinement alone (without any attraction between the liquid molecules and the surface) could explain the layered structure of liquids at the interface. We rationalize the layering by the reduced excluded volume of molecules close to a surface. With MD simulations we find, however, that for the specific case of the graphite-water interface at ambient conditions the confinement alone is not the origin of the solvation layers. Our result highlights the significance of the attractive water-graphite interaction since the attraction is key for the formation of hydration layers.

\section{OBSERVATION OF HYDRATION LAYERS AT THE GRAPHITE-WATER INTERFACE}

We performed 3D AFM experiments to map the hydration structure at the graphite-water interface. Experimental details can be found in Appendix A. A representative vertical slice of a 3D data set in Fig. 1 shows the excitation frequency shift. The slice does not show any lateral structure, which is in agreement with previously recorded two-dimensional (2D) AFM data by Suzuki et al. [8]. However, several

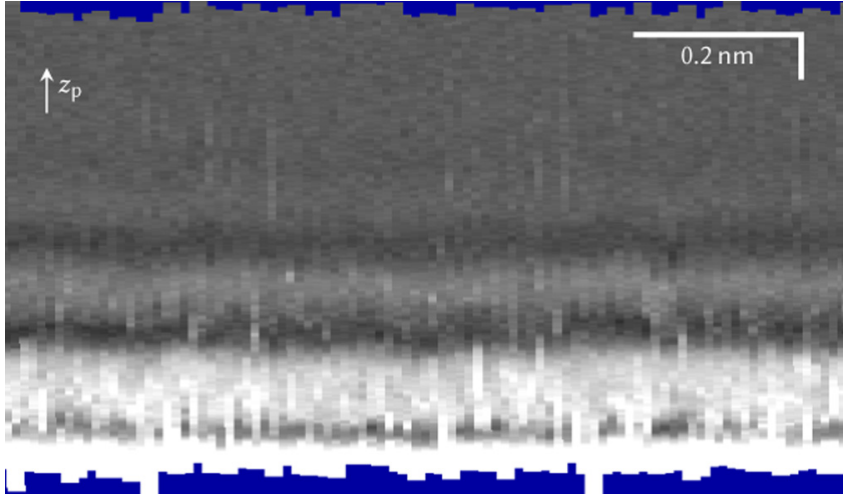

FIG. 1. A vertical slice of 3D AFM data showing the excitation frequency shift obtained at the graphite-water interface. The color scale ranges from -7 to $13 \mathrm{kHz}$.

vertical layers with a layer-to-layer distance in the order of $0.4 \mathrm{~nm}$ are visible at the interface. When using the solvent tip approximation $[38,39]$ according to the discussion in Ref. [7], the local maxima in the excitation frequency shift are assigned to local maxima in the water density. These experimental results, along with the large number of research papers describing the observation of layers at interfaces, raise the fundamental question as to whether the observed solvation layers are indeed caused solely by the confining nature of the surface or whether they appear because of attractive interactions between the graphite surface and water (or due to a combination of both).

\section{SOLVATION LAYERS CAUSED BY CONFINEMENT ALONE}

In this section, we address the question as to whether confinement alone, without attraction, can explain the observed layering at solid-liquid interfaces. For this, we performed a MC simulation [40] (technical details can be found in Appendix B) of a most simple solid-liquid interface: A onedimensional model system of hard spheres (representing the liquid molecules), confined between two hard walls representing two solid surfaces. This model assumes zero force between the hard spheres and between the spheres and the walls, i.e., there is no attraction between the liquid molecules and the surface.

We denote the sphere radius as $\sigma$ and choose a wall-to-wall distance of $L=40 \sigma$ (i.e., at maximum 20 spheres can be placed in the system). Details on the MC simulation can be found in Appendix B. In Fig. 2(a), we plot the simulated probability density $p$ of finding a sphere at position $z$ between the two walls. The different curves show the probability densities corresponding to systems containing one sphere to 15 spheres.

If one sphere is between the two walls, the probability of finding the sphere at a possible position is constant (curve indicated with " 1 ," also shown in the inset). For $z<\sigma$ and $z>L-\sigma$, the probability is zero since wall and spheres are impenetrable. This is indicated by the red area in Fig. 2(b), in which the (left) wall is depicted by the block (since the system is symmetric, the right half is not shown). In this case, no layers are formed. 


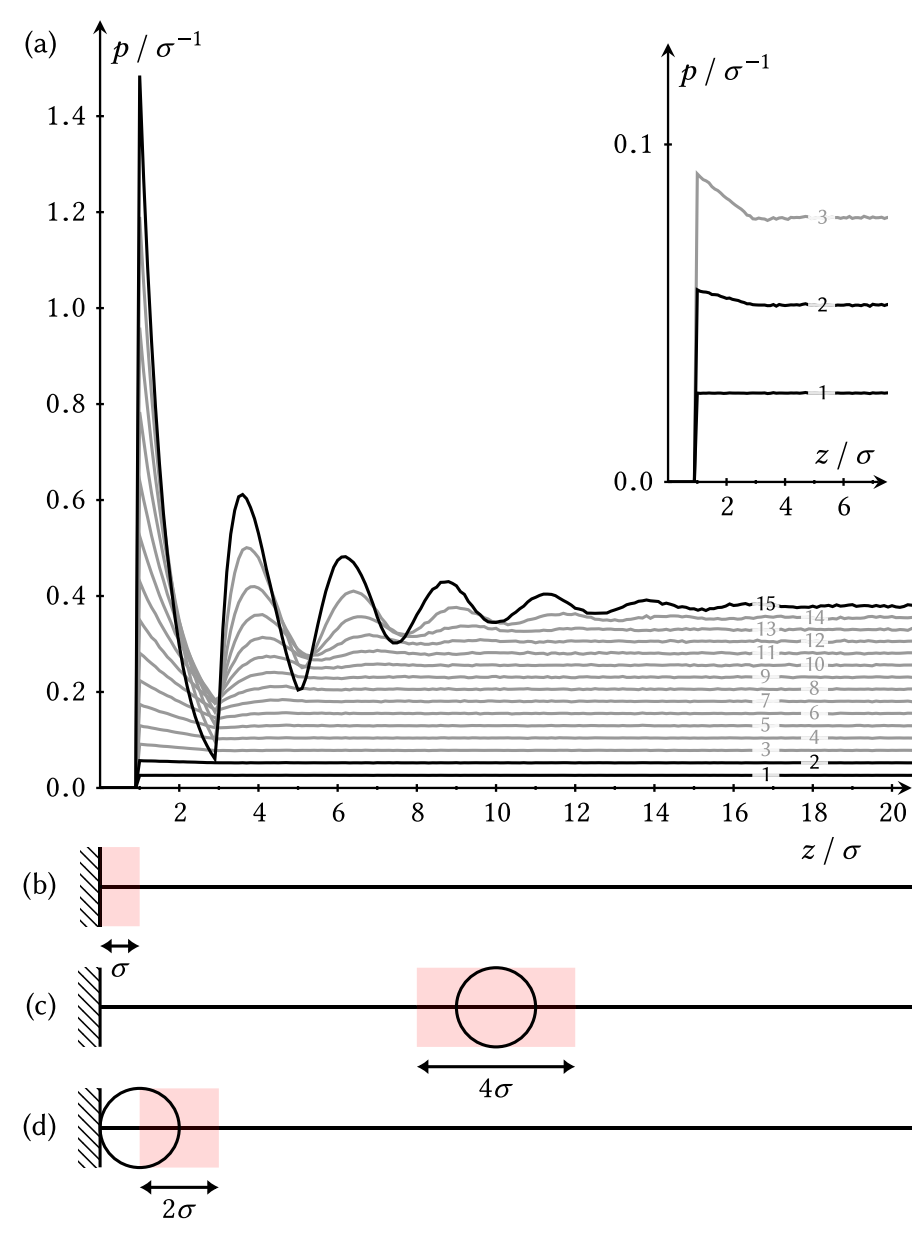

FIG. 2. MC simulation of a one-dimensional system of hard spheres with radius $\sigma$ between two hard walls spaced $40 \sigma$ apart. In (a), the probability density $p$ of finding a sphere is plotted as function of the position $z$ between the two walls. The different curves show the probability densities for one to 15 spheres in the system. The space blocked by the walls is indicated by the shaded area in (b). In (c), (d), the space blocked by a single sphere placed far away and close to the wall, respectively, is indicated.

Already when placing two spheres between the two walls, a deviation from the uniform distribution can be recognized (curve indicated with "2" in Fig. 2, also shown in the inset). For a two-sphere system, the probability of placing the second sphere at position $z$ is equal to the probability of the first sphere not blocking position $z$. Therefore, finding the second sphere at any given position depends on the position of the first sphere. If the first sphere is placed far away from the wall, this sphere blocks a space of $4 \sigma$ for the position of the second sphere [Fig. 2(c)]. However, if the first sphere is placed directly at the wall, it only blocks an additional space of $2 \sigma$ on top of the space blocked by the wall [Fig. 2(d)]. Thus, any sphere placed close to a wall excludes a smaller space compared to placing it away from the wall. Consequently, the space away from the walls is more likely blocked. Therefore, the probability of finding the second sphere close to the wall is increased.

When considering more spheres between the walls, the accumulation of spheres directly at the interface becomes even more pronounced (peak at $z=\sigma$ in Fig. 2). This first layer can be considered as an additional effective wall. Therefore, the first layer confines the spheres further away from the wall and causes the formation of a second layer. Consequently, a pronounced layered structure emerges when increasing the number of spheres. In the extreme case of placing the maximum number of spheres between the walls, the liquid is perfectly structured. This discussion concludes that layering can be obtained in the simplified case of a one-dimensional hard sphere liquid with hard walls, even without attractive interactions. From the simulation we conclude that it is at least possible that the observed water layering at the graphite surface can be explained solely by the confinement. This is in line with many previous research papers stated in the introduction. In summary, confinement causes layers since molecules close to the wall block less space. This argument holds regardless of considering a one-dimensional or a three-dimensional system. Next, we check how attractive molecule-molecule interactions influence the interfacial layers.

\section{MOLECULE-MOLECULE ATTRACTION SUPPRESSES HYDRATION LAYERS}

In this section, we extend our MC simulations by including attractive interactions between the hard spheres. For simplicity, we model the sphere-sphere attraction with a potential energy of $-E_{\mathrm{SS}} / d$, where $d \geqslant 2 \sigma$ is the distance between the center of two spheres and $E_{\mathrm{SS}}>0$ quantifies the strength of the attraction. As before, spheres cannot penetrate each other. The corresponding density profiles originating from a simulation with varying degrees of attraction are shown in Fig. 3.

The space directly adjacent to the wall $(0<z<\sigma)$ again cannot be occupied by any sphere. At larger distances from the wall $(z>\sigma)$, the overall magnitude of the oscillating density profile decreases when increasing the sphere-sphere attraction. This can be readily explained by the number of neighbors of each sphere. A sphere close to the wall $(\sigma<z<3 \sigma)$ has

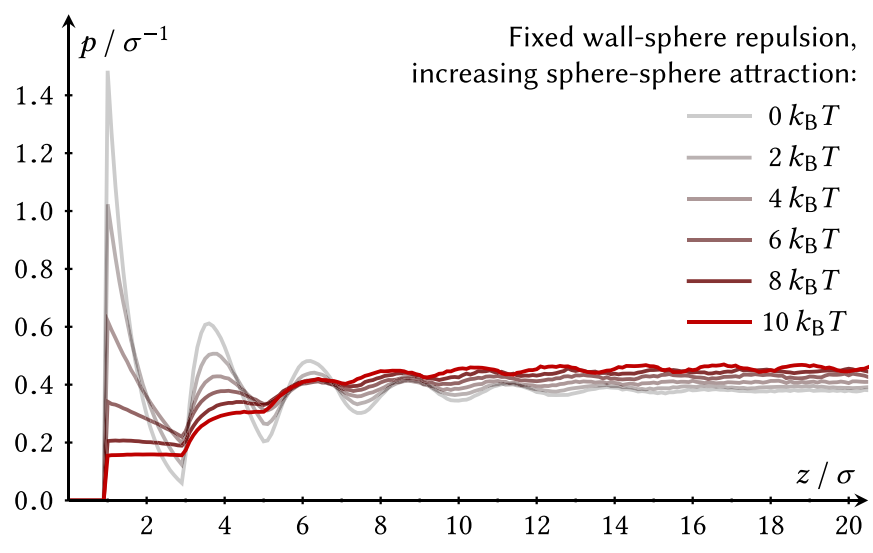

FIG. 3. MC simulation of a one-dimensional system of spheres between two walls. The sphere-wall interaction is modeled with a purely repulsive hard-wall potential. The sphere-sphere interaction is modeled with an attractive interaction according to $-E_{\mathrm{SS}} / d$, where $d$ is the distance between two spheres and $E_{\mathrm{SS}}$ varies from zero (no attraction, black half-transparent line) to $10 k_{\mathrm{B}} T$ (red line). 


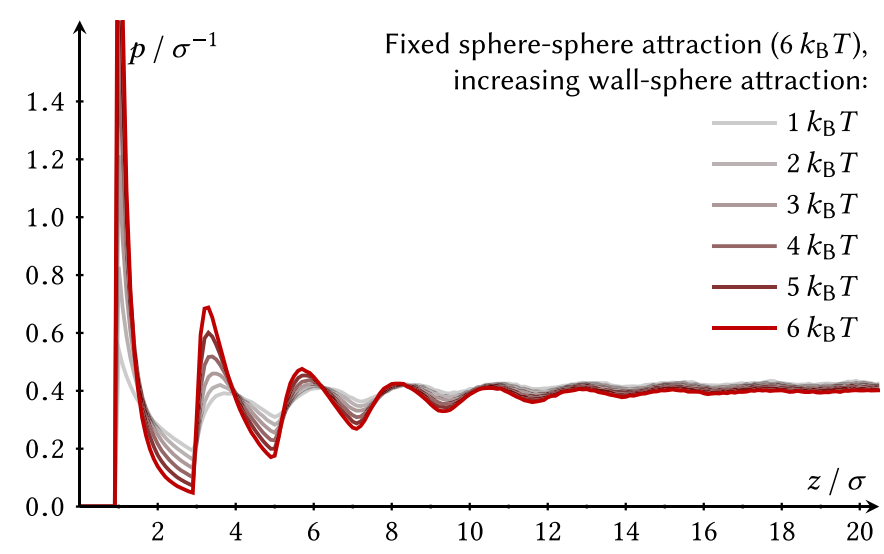

FIG. 4. Density profiles from MC simulations with attractive forces acting between the spheres and between the spheres and the wall. The sphere-wall interaction is modeled with a potential of $-E_{\mathrm{SW}} / d$ that is superimposed on a hard-wall potential. The factor $E_{\mathrm{SW}}$ varies from $k_{\mathrm{B}} T$ (black half-transparent line) to $6 k_{\mathrm{B}} T$ (red line).

only neighboring spheres to its right (at larger $z$ ). Since the spheres interact attractively with each other, a position close to the wall becomes increasingly unfavorable when increasing the sphere-sphere attraction. Positions further away from the wall are favored because molecules far away from the wall are, on average, surrounded by more molecules compared to molecules close to the wall. Figure 3 reveals that this effect results in an increasing probability density for increasing separation from the wall, in particular when considering a large attractive interaction (red curve). Increasing sphere-sphere attraction causes the layering to gradually diminish.

\section{SOLVATION LAYERS INDUCED BY MOLECULE-WALL ATTRACTION}

Next, we change the wall-sphere interaction in the MC simulation by considering an interaction potential of $-E_{\mathrm{SW}} / d$ between each sphere and each wall. Here, $E_{\mathrm{SW}}>0$ quantifies the sphere-wall attraction and $d \geqslant \sigma$ is the distance between the sphere and the wall. As before, the spheres cannot penetrate the wall and the sphere-sphere interaction is modeled as described in the previous section.

The results are shown in Fig. 4 for a fixed sphere-sphere attraction of $6 k_{\mathrm{B}} T$ and an increasing wall-sphere attraction. A pronounced layering is again visible for an increasing wallsphere attraction. This finding is reasonable since the wall effectively reduces the energy penalty that was previously associated with the smaller number of neighboring spheres close to the wall. ${ }^{1}$

Our results demonstrate that the interplay between molecule-molecule and molecule-surface interactions dictates the overall manifestation of the layers by confinement. Both confinement (Fig. 2) and attractive molecule-surface interactions (Fig. 4) can cause the formation of layers. An attractive molecule-molecule interaction can cause the layers to vanish

\footnotetext{
${ }^{1}$ Note that the case $E_{\mathrm{SS}}>0$ and $E_{\mathrm{SS}}=E_{\mathrm{SW}}$ does not result in the same density distribution compared to the case where both $E_{\mathrm{SS}}$ and $E_{\mathrm{SW}}$ are zero.
}
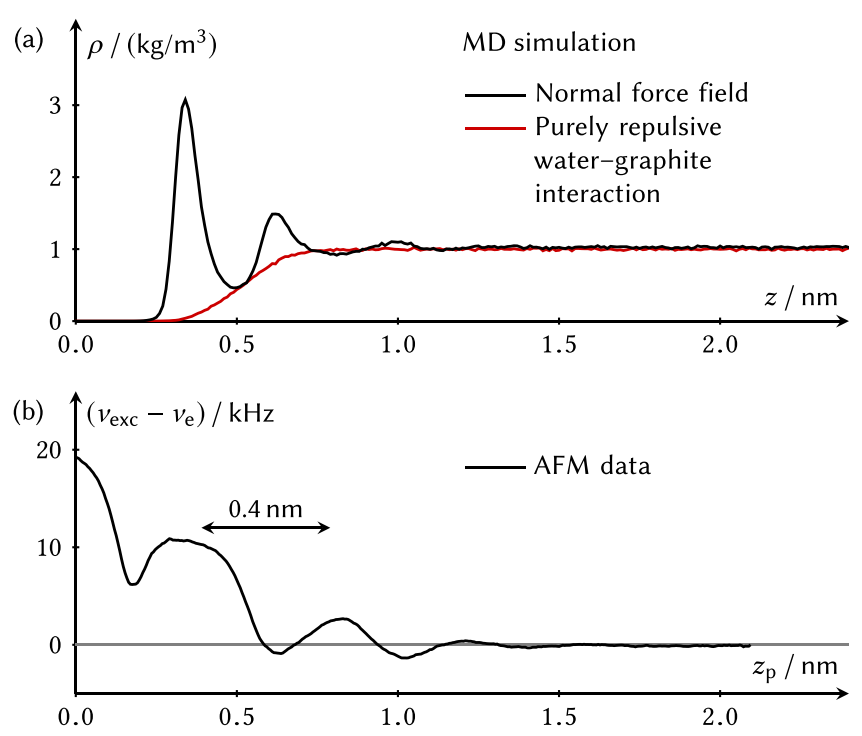

FIG. 5. Comparison of MD simulation results (a) with the experimental AFM data (b). In (a), water-oxygen density profiles (normal to the surface, in $z$ direction) are shown for a force field with attraction (black curve) and for the purely repulsive carbon-water interaction (red curve). The vertical average over all experimental AFM data shown in Fig. 1 as function of the $z$-piezo displacement $z_{\mathrm{p}}$ is shown in (b) for a direct comparison with the MD data. The offsets of the horizontal axes of (a) and (b) are arbitrarily aligned.

(Fig. 3). Thus, the observation of solvation layers at interfaces by itself does not allow to conclude whether the layers appear due to confinement, attraction, or a combination of both.

\section{GRAPHITE-WATER INTERFACE AT AMBIENT CONDITIONS: ATTRACTION OR CONFINEMENT?}

Next, we present MD simulations to clarify the origin of the observed hydration layers for the specific case of the graphite-water interface at ambient conditions (and, in contrast to the previous sections, considering three spatial dimensions). First, we analyze density profiles (Fig. 5) of water equilibrated at ambient conditions ( $300 \mathrm{~K}$ and $1013 \mathrm{hPa}$ ) at the graphite-water interface, considering both a commonly used force field (described briefly below and in Appendix C) as well as a force field where we do not include the attractive contribution to the graphite-water interaction.

The first force field we employ is one of the commonly used force fields developed for interactions between water and graphite [41]. The resulting density profile [black line in Fig. 5(a)] exhibits distinct peaks corresponding to hydration layers. The simulation results fit to the experimental 3D AFM data [Fig. 1, shown as an averaged vertical profile in Fig. 5(b)] which shows layers as well.

Next, we remove the attractive contribution from the carbon-water interaction within the MD simulation [Fig. 5(a), red curve] to test whether the graphite-water attraction is necessary for the hydration layer formation or whether the confinement alone causes the formation of layers in this case. In sharp contrast to the previous case, we observe no hydration layers (at ambient conditions) in this case. The region close to the surface where previously the first two hydration layers 
have been is now depleted of liquid. Instead of an oscillatory density profile, the density increases monotonically (as is consistent with previous works [34-36]).

While it is expected that the layers diminish when removing the attractive contribution from the graphite-water interaction, it is crucial to note that the layers indeed vanish completely, thereby showing that the confinement alone does not suffice to explain the layering. The monotonic density increase without oscillations obtained at the repulsion-only graphite-water interface can be interpreted as being dominated by the water-water attraction (compare Fig. 3). We thereby demonstrate that the confining graphite surface alone cannot cause hydration layers due to the strong water-water attraction. The graphite-water attraction is, therefore, key for the formation of hydration layers.

\section{CONCLUSION}

Three-dimensional AFM data show that water is arranged in layers above the graphite surface. In this paper, we answer the question whether these hydration layers are caused by graphite-water attraction or by confinement due to the presence of the surface.

Based on MC simulations of a most simple hard-sphere liquid between two walls, we explain why, in principle, layers can arise purely due to the surface confinement, even in the absence of attraction between wall and liquid. A sufficiently large intermolecular attraction between the liquid can, however, cause the layers to diminish, while attraction between liquid and wall has the opposite effect. From this analysis, we conclude that the presence of layers does not allow to differentiate between attraction or confinement as their physical origin.

We performed MD simulations of the graphite-water interface which demonstrate that layers only occur when the attractive contribution of the graphite-water interaction is included as, otherwise, no layers form. Thus, even though confinement can induce hydration layers, for the specific case of graphite, a significant graphite-water attraction is key for the hydration layers to form.

This work demonstrates that a clear disentanglement of attraction and confinement is required when aiming for understanding layer formation at solid-liquid interfaces.

\section{ACKNOWLEDGMENTS}

We are grateful to P. Raiteri for significantly improving the manuscript by his many critical suggestions. We gratefully acknowledge financial support from the German Research Foundation (DFG) through Grant No. KU 1980/7-1. Y.M.J. and A.S.F. were supported by the Academy of Finland (project no. 314862)

\section{APPENDIX A: 3D AFM EXPERIMENTS}

The graphite (0001) surface (PLANO GmbH, Germany) was freshly cleaved with adhesive tape in air and cleaned under a nitrogen flow prior to the measurement. All AFM measurements were performed in pure water (Millipore $\mathrm{GmbH}$, Germany) with a custom 3D AFM in the frequency- modulation mode [42-44]. An O-ring was used to prevent water evaporation. Oscillation amplitudes in the order of 0.05 to $0.1 \mathrm{~nm}$ were used. We used silicon cantilevers (TAP 300GD-G, BudgetSensors, Bulgaria) with an eigenfrequency of 100 to $150 \mathrm{kHz}$, a quality factor of approximately 7 in water and a nominal spring constant of approximately $40 \mathrm{Nm}^{-1}$.

\section{APPENDIX B: MC SIMULATIONS}

The simulation steps of the MC simulation were performed as follows: First, a random sphere is selected. This sphere is then placed at a random position within the two walls. Next, the total potential energy of the system $E$ is calculated by summing up all wall-sphere interaction energies and all contributions due to sphere-sphere interactions. The hard-wall potential is described by an infinite potential energy when a sphere overlaps the wall or another sphere and, for all other positions, a potential energy contribution of zero. Next, we select a random number $r \in[0,1]$ and accept the sphere move if $r \leqslant \exp \left[-\Delta E /\left(k_{\mathrm{B}} T\right)\right]$, where $\Delta E$ is the energy change due to the move. The Boltzmann constant $k_{\mathrm{B}}$ and temperature $T$ are canceled in the simulation as we supply the interaction energy in units of $k_{\mathrm{B}} T$ (Fig. 3). This process is repeated until $10^{8}$ sphere moves have been performed. The first $10^{5}$ steps of the simulation are discarded to eliminate any influence of the starting configuration. After each accepted step, a histogram of the sphere positions was recorded. After the simulation, the histograms were averaged to obtain the probability density $p(z)$ of finding a sphere at position $z$. The value of the integral of the probability density $p$ from $z=0$ to $40 \sigma$ equals the number of spheres between the two walls.

\section{APPENDIX C: MD SIMULATIONS}

\section{Setup}

For our MD simulations we used the LAMMPS code [45]. The simulation systems consist of graphite slabs (with eight graphene layers in total) stacked in an $\mathrm{AB}$ configuration and water slabs with 1000, 2000, 3000, and 4000 water molecules, respectively. In Fig. 6 we show the simulation system for 4000 water molecules (already after the equilibration described below). We centered the water in the middle of the box and placed graphite slabs on its top and bottom. The surface area of graphite is about $5 \mathrm{~nm} \times 5 \mathrm{~nm}$ and the slab thickness is about $1 \mathrm{~nm}$ for each slab. Periodic boundary conditions were used in all directions.

The simulation procedure consisted of an energy minimization with conjugated gradients, 500 ps NVT equilibration at $300 \mathrm{~K}, 500 \mathrm{ps}$ NPT equilibration at $300 \mathrm{~K}$ and $1013 \mathrm{hPa}$, and then a production run of 2000 ps in NVT ensemble (with a time step of 0.001 ps for all simulations). We used Nosé-Hoover thermostats and barostats $[46,47]$. For simulations where we decreased the distance between graphite slabs (maintaining the same number of water molecules) we fixed the $z$ coordinate (normal to the surface) of the carbon atoms, but let the $x$ and $y$ coordinates unconstrained. Analysis was performed with MDAnalysis [48,49].

Since the experiments were performed at ambient conditions, we kept the pressure of the MD simulation constant at $1013 \mathrm{hPa}$ (unless otherwise stated). Note that we did not include an AFM cantilever tip in the simulations, thereby also 


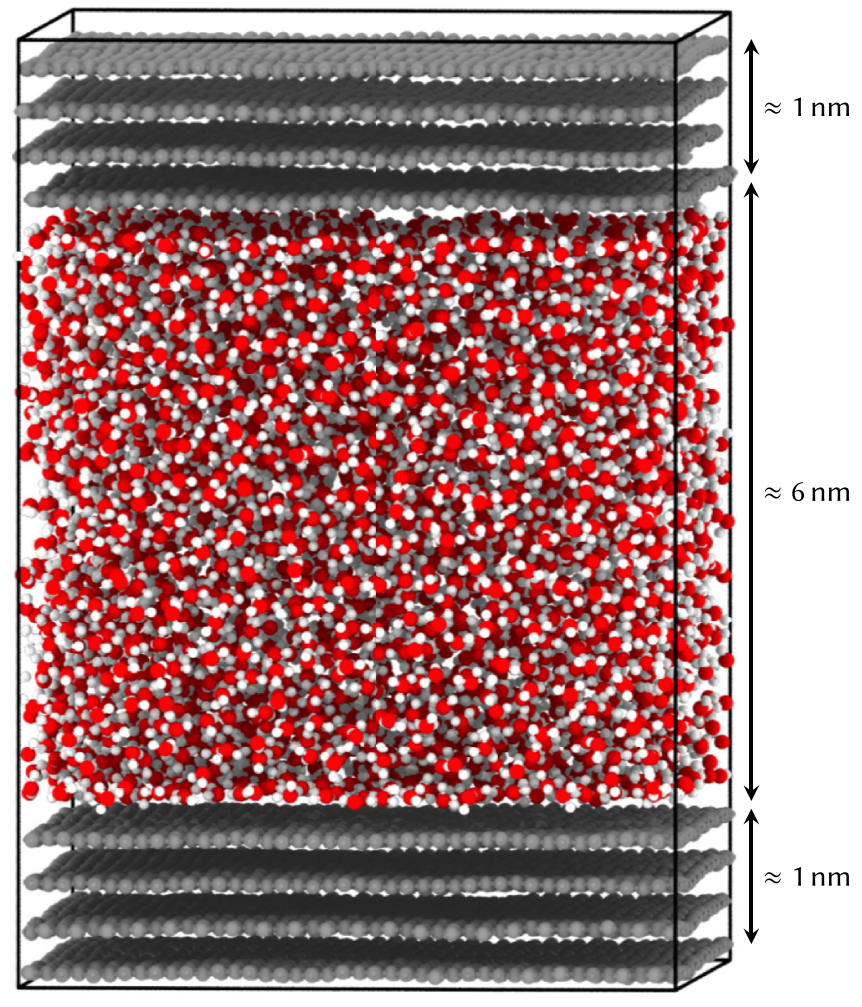

FIG. 6. Setup of the MD simulation box used for the data presented in Fig. 5(a) as the black curve (the simulation with the full force field by Wu and Aluru [41]). The simulation frame corresponds to the equilibrated system.

neglecting a possible tip-induced local pressure change at the interface. We rationalize this by the vastly different timescales of the AFM tip oscillation and the dynamics of water in the simulation.

\section{Force fields}

We used the SPC/Fw flexible model for water molecules [50] and the AIREBO interatomic potential [51] for carbon atoms. For the interactions between carbon and water, we used different parametrizations: In the simulations where we included the full Lennard-Jones potentials for the carbon-water interaction (with both attractive and repulsive contributions) we used the standard Lennard-Jones potential

$$
E=4 \epsilon\left[\left(\frac{\sigma}{r}\right)^{12}-\left(\frac{\sigma}{r}\right)^{6}\right]
$$

where we set $\epsilon$ and $\sigma$ for the $\mathrm{C}-\mathrm{O}$ and $\mathrm{C}-\mathrm{H}$ interactions. We took the parametrizations for the simulations from $\mathrm{Wu}$ and Aluru [41] and additionally show in this Appendix the result obtained from the parametrization by Werder et al. [52].

For the purely repulsive carbon-water interaction we used the A/B form of the Lennard-Jones potential in LAMMPS:

$$
E=\frac{A}{r^{12}}-\frac{B}{r^{6}} .
$$

We used $\epsilon$ and $\sigma$ from the Werder et al. [52] parametrization to set $A=4 \epsilon \sigma^{12}$ and $B$ to zero (thus disabling the attractive part of the potential).

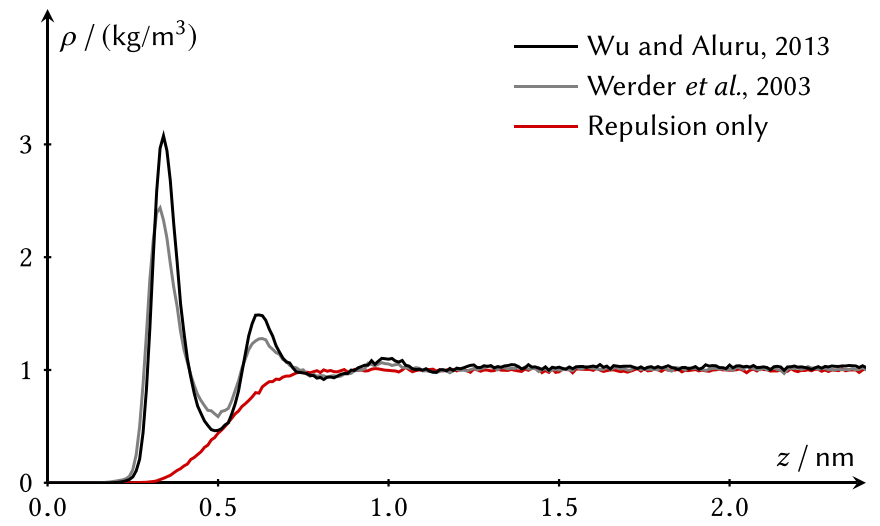

FIG. 7. Water-oxygen density profiles perpendicular to the surface for the Wu and Aluru [41] parametrization [black curve, same as in Fig. 5(a)] and the parametrization from Werder et al. [52]. The results for the purely repulsive carbon-water interaction are shown in red [same as in Fig. 5(a)].

A comparison of density profiles from all three simulations is shown in Fig. 7. Our results are consistent with previous works [34-36], which report diminishing layers for vanishing attractive contributions as well.

A direct comparison of the $\mathrm{Wu}$ and Aluru [41] with the Werder et al. [52] parametrization shows that the curves are qualitatively similar. However, the Werder et al. parametrization results in comparatively smaller water density within the layers. When interpreting contact angles as an indication of the solid-liquid interaction strength at the interface (if all other parameters are equal), the observed trend in the density curves fits to the simulated water contact angles of about $40^{\circ}$ for the $\mathrm{Wu}$ and Aluru parametrization [41] and about $86^{\circ}$ obtained for the Werder et al. [52] parametrization.

Note that the curve resulting from the MC simulation with the largest sphere-sphere attraction considered in Fig. 3 matches qualitatively with the MD simulation data [red curve in Fig. 5(a) and in Fig. 7] in that it shows a monotonic, nonoscillatory density increase close to the interface. The steplike features in the density profile of the MC simulations originate from the fact that only hard spheres within a onedimensional system have been considered. In contrast, the $3 \mathrm{D}$ system used in the MD simulation allows to average over several different surface sites which effectively leads to "smoother" average density curves. The additional oscillatory behavior that can be recognized in the center of the MC simulation box originates from a dense packing of the spheres.

\section{Additional simulations}

Our major conclusion from the MD simulations presented in the main text is that at ambient conditions, the confining nature of the surface alone does not cause hydration layers to form at the graphite-water interface. We performed further simulations with the purely repulsive carbon-water interaction to verify the robustness of this statement.

First, we decreased the number of water molecules between the slabs to values between 3000 and 1000 (originally we had 4000 water molecules). Each of these systems was equilibrated as NVT ensemble followed by NPT simulations in all directions to let the systems reach the correct density. 


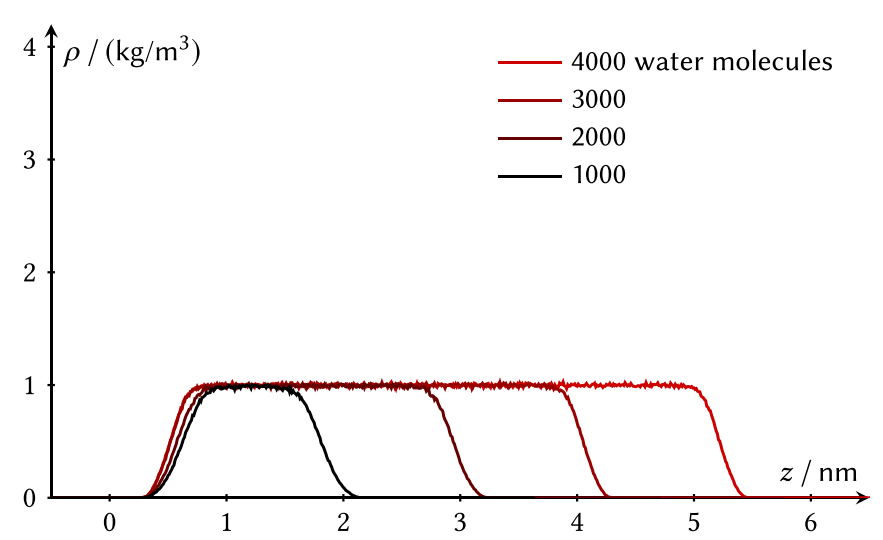

FIG. 8. Water-oxygen density profiles perpendicular to the surface for the purely repulsive carbon-water interaction and a decreasing amount of water.

This ultimately resulted in a smaller distance between the graphite slabs.

The density profiles for those systems are shown in Fig. 8. There is no qualitative change in the density profiles as the spacing decreases. This shows that at normal pressure $(1013 \mathrm{hPa})$ no hydration layers are formed at the graphitewater interface when only repulsive interactions are considered, even when the liquid is confined to a space as small as $2.5 \mathrm{~nm}$.

Second, we reduced the spacing between graphite slabs while maintaining the original number of water molecules between them. This results in a compression of the system. To study the system in this configuration, we restrained the movement in the $z$ direction of the carbon atoms, while allowing them (as well as the simulation box) to move in $x$ and $y$.

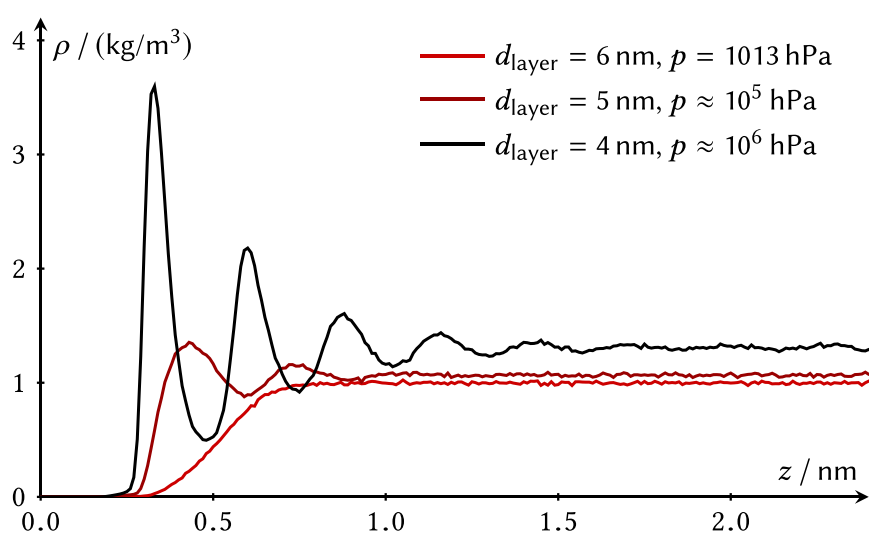

FIG. 9. Water-oxygen density profiles perpendicular to the surface for the purely repulsive carbon-water interaction and decreasing slab spacings of 6,5 , and $4 \mathrm{~nm}$.

Density profiles for those configurations are shown in Fig. 9. As we compress the water slab, hydration layers are eventually formed, even though the interactions between solid and liquid are purely repulsive (similar to the MC simulation presented in Sec. II).

These results are in accordance with a recent study [53] where it was found that increased pressure led to formation or strengthening of hydration layers between rigid graphene sheets with standard Lennard-Jones potentials.

However, in our simulations we observed that the reduction of the graphite-graphite distance from $6 \mathrm{~nm}$ (with a pressure of $1013 \mathrm{hPa}$ ) to 5 and $4 \mathrm{~nm}$ yielded comparatively large pressures within the order of $10^{5}$ to $10^{6} \mathrm{hPa}$, respectively. In accordance, the bulk density increases from $1 \mathrm{~g} \mathrm{~cm}^{-3}$ up to approximately $1.3 \mathrm{~g} \mathrm{~cm}^{-3}$. Thus, these systems cannot be considered to describe the interface at ambient conditions.
[1] Y. Nagata, K. Usui, and M. Bonn, Phys. Rev. Lett. 115, 236102 (2015).

[2] M. A. Sánchez, T. Kling, T. Ishiyama, M.-J. van Zadel, P. J. Bisson, M. Mezger, M. N. Jochum, J. D. Cyran, W. J. Smit, H. J. Bakker, M. J. Shultz, A. Morita, D. Donadio, Y. Nagata, M. Bonn, and E. H. G. Backus, Proc. Natl. Acad. Sci. USA 114, 227 (2016).

[3] O. M. Magnussen and A. Groß, J. Am. Chem. Soc. 141, 4777 (2019).

[4] T. Fukuma and R. Garcia, ACS Nano 12, 11785 (2018).

[5] T. Fukuma, Y. Ueda, S. Yoshioka, and H. Asakawa, Phys. Rev. Lett. 104, 016101 (2010).

[6] D. Martin-Jimenez, E. Chacon, P. Tarazona, and R. Garcia, Nat. Commun. 7, 12164 (2016).

[7] H. Söngen, C. Marutschke, P. Spijker, E. Holmgren, I. Hermes, R. Bechstein, S. Klassen, J. Tracey, A. S. Foster, and A. Kühnle, Langmuir 33, 125 (2017).

[8] K. Suzuki, N. Oyabu, K. Kobayashi, K. Matsushige, and H. Yamada, Appl. Phys. Express 4, 125102 (2011).
[9] T. Utsunomiya, Y. Yokota, T. Enoki, and K. Fukui, Chem. Commun. 50, 15537 (2014).

[10] T. Utsunomiya, S. Tatsumi, Y. Yokota, and K. Fukui, Phys. Chem. Chem. Phys. 17, 12616 (2015).

[11] L. Cheng, P. Fenter, K. L. Nagy, M. L. Schlegel, and N. C. Sturchio, Phys. Rev. Lett. 87, 156103 (2001).

[12] P. Geissbühler, P. Fenter, E. DiMasi, G. Srajer, L. B. Sorensen, and N. C. Sturchio, Surf. Sci. 573, 191 (2004).

[13] S. Pintea, W. de Poel, A. E. F. de Jong, V. Vonk, P. van der Asdonk, J. Drnec, O. Balmes, H. Isern, T. Dufrane, R. Felici, and E. Vlieg, Langmuir 32, 12955 (2016).

[14] R. G. Horn and J. N. Israelachvili, J. Chem. Phys. 75, 1400 (1981).

[15] J. N. Israelachvili and R. M. Pashley, Nature (London) 306, 249 (1983).

[16] J. Israelachvili and H. Wennerström, Nature (London) 379, 219 (1996).

[17] J. N. Israelachvili, Intermolecular and Surface Forces, 3rd ed. (Elsevier, Amsterdam, 2011). 
[18] M. E. Fisher and B. Widom, J. Chem. Phys. 50, 3756 (1969).

[19] F. F. Abraham, J. Chem. Phys. 63, 157 (1975).

[20] F. F. Abraham, J. Chem. Phys. 68, 3713 (1978).

[21] F. F. Abraham and Y. Singh, J. Chem. Phys. 67, 2384 (1977).

[22] F. F. Abraham and Y. Singh, J. Chem. Phys. 68, 4767 (1978).

[23] D. Henderson, F. F. Abraham, and J. A. Barker, Mol. Phys. 31, 1291 (1976).

[24] J. R. Henderson, Mol. Phys. 59, 89 (1986).

[25] I. K. Snook and D. Henderson, J. Chem. Phys. 68, 2134 (1978).

[26] I. K. Snook and W. van Megen, J. Chem. Phys. 70, 3099 (1979).

[27] I. K. Snook and W. van Megen, J. Chem. Phys. 72, 2907 (1980).

[28] W. van Megen and I. Snook, J. Chem. Soc., Faraday Trans. 75, 1095 (1979).

[29] W. J. van Megen and I. K. Snook, J. Chem. Phys. 74, 1409 (1981).

[30] J. D. Weeks, D. Chandler, and H. C. Andersen, J. Chem. Phys. 54, 5237 (1971).

[31] P. Kumar, S. V. Buldyrev, F. W. Starr, N. Giovambattista, and H. E. Stanley, Phys. Rev. E 72, 051503 (2005).

[32] P. Kumar, F. W. Starr, S. V. Buldyrev, and H. E. Stanley, Phys. Rev. E 75, 011202 (2007).

[33] L. B. Krott and M. C. Barbosa, Phys. Rev. E 89, 012110 (2014).

[34] A. P. Willard and D. Chandler, J. Chem. Phys. 141, 18C519 (2014).

[35] N. Choudhury, Chem. Phys. 421, 68 (2013).

[36] F. Mozaffari, Mol. Simul. 42, 1475 (2016).

[37] A. Kozbial, F. Zhou, Z. Li, H. Liu, and L. Li, Acc. Chem. Res. 49, 2765 (2016).

[38] M. Watkins and B. Reischl, J. Chem. Phys. 138, 154703 (2013).
[39] K. Amano, K. Suzuki, T. Fukuma, O. Takahashi, and H. Onishi, J. Chem. Phys. 139, 224710 (2013).

[40] N. Metropolis, A. W. Rosenbluth, M. N. Rosenbluth, A. H. Teller, and E. Teller, J. Chem. Phys. 21, 1087 (1953).

[41] Y. Wu and N. R. Aluru, J. Phys. Chem. B 117, 8802 (2013).

[42] S. Rode, R. Stark, J. Lübbe, L. Tröger, J. Schütte, K. Umeda, K. Kobayashi, H. Yamada, and A. Kühnle, Rev. Sci. Instrum. 82, 073703 (2011).

[43] H. Adam, S. Rode, M. Schreiber, K. Kobayashi, H. Yamada, and A. Kühnle, Rev. Sci. Instrum. 85, 023703 (2014).

[44] H. Söngen, M. Nalbach, H. Adam, and A. Kühnle, Rev. Sci. Instrum. 87, 063704 (2016).

[45] S. Plimpton, J. Comput. Phys. 117, 1 (1995).

[46] S. Nosé, J. Chem. Phys. 81, 511 (1984).

[47] W. G. Hoover, Phys. Rev. A 31, 1695 (1985).

[48] R. J. Gowersk, M. Linke, J. Barnoud, T. J. E. Reddy, M. N. Melo, S. L. Seyler, J. Domanski, D. L. Dotson, S. Buchoux, I. M. Kenney, and O. Beckstein, in Proceedings of the 15th Python in Science Conference, edited by S. Benthall and S. Rostrup (2016), pp. 98-105.

[49] N. Michaud-Agrawal, E. J. Denning, T. B. Woolf, and O. Beckstein, J. Comput. Chem. 32, 2319 (2011).

[50] Y. Wu, H. L. Tepper, and G. A. Voth, J. Chem. Phys. 124, 024503 (2006).

[51] S. J. Stuart, A. B. Tutein, and J. A. Harrison, J. Chem. Phys. 112, 6472 (2000).

[52] T. Werder, J. H. Walther, R. L. Jaffe, T. Halicioglu, and P. Koumoutsakos, J. Phys. Chem. B 107, 1345 (2003).

[53] T. Samanta, R. Biswas, S. Banerjee, and B. Bagchi, J. Chem. Phys. 149, 044502 (2018). 